\title{
Data Prediction in Distributed Sensor Networks Using Adam Bashforth Moulton Method
}

\author{
Md Monirul Islam, Zabir Al Nazi, A. B. M. Aowlad Hossain, Md Masud Rana \\ Department of Electronics and Communication Engineering, Khulna University of Engineering \\ and Technology, Khulna, Bangladesh \\ Email:mohammad.monirul.islam.10@gmail.com, zabiralnazi@codeassign.com, aowlad0403@yahoo.com
}

How to cite this paper: Islam, M.M., Al Nazi, Z., Hossain, A.B.M.A. and Rana, M.M. (2018) Data Prediction in Distributed Sensor Networks Using Adam Bashforth Moulton Method. Journal of Sensor Technology, 8, 48-57.

https://doi.org/10.4236/jst.2018.82004

Received: September 27, 2017

Accepted: June 18, 2018

Published: June 21, 2018

Copyright () 2018 by authors and Scientific Research Publishing Inc. This work is licensed under the Creative Commons Attribution International License (CC BY 4.0).

http://creativecommons.org/licenses/by/4.0/

\begin{abstract}
Information collection from remote location is very important for several tasks such as temperate monitoring, air quality investigation, and wartime surveillance. Wireless sensor network is the first choice to complete these types of tasks. Basically, information prediction scheme is an important feature in any sensor nodes. The efficiency of the sensor network can be improved to large extent with a suitable information prediction scheme. Previously, there were several efforts to resolve this problem, but their accuracy is decreased as the prediction threshold reduces to a small value. Our proposed Adams-Bashforth-Moulton algorithm to overcome this drawback was compared with the Milne Simpson scheme. The proposed algorithm is simulated on distributed sensor nodes where information is gathered from the Intel Berkeley Research Laboratory. To maximize the power saving in wireless sensor network, our adopted method achieves the accuracy of 60.28 and 59.2238 for prediction threshold of 0.01 for Milne Simpson and Adams-Bashforth-Moulton algorithms, respectively.
\end{abstract}

\section{Keywords}

Adams-Bashforth-Moulton Method, Energy Saving, Milne-Simpson Method, Wireless Sensor Networks

\section{Introduction}

A large number of sensors are deployed in the physical environment to collect sensing information, and this network is known as wireless sensor network (WSN). In real-time, the sensors which are utilised in physical environments have low power and low computation capability. Consequently, with scarce facility, a sensor has to fulfill these essential tasks. Usually, the sensor network is 
divided into many sub-networks also known as cluster heads [1] [2] [3] [4]. Each cluster head has numerous nodes. Generally speaking, the cluster heads are connected to both sensor nodes and base stations. The connection of cluster head and sensor node can be repeated again and again for very large-scale WSN. When a node sends information, it is transmitted to the cluster head and via the cluster. Then the cluster head information is transmitted to the base station where further processing is performed.

The higher the accuracy of successful data prediction, the better energy efficient sensor network is. Generally speaking, when information is gathered on continuous fashion, the energy required for transmitting all these bits will incur most of the energy of cluster heads, making the system energy inefficient. In order to solve this drawback, we adopted a method which can reduce information transmission rate by predicting more accurate information. As the prediction accuracy increases, the necessity of transmitting information is not required, resulting in low power consumption. In this way, the sensor node can save energy which is very necessary in a tiny device. Driven by this motivation, this paper proposes a Adams-Bashforth-Moulton scheme for information prediction and correction in the context of distributed WSN.

The main contribution of this work is to propose Adams-Bashforth-Moulton scheme for information prediction and correction. First of all, sensors are used to sense information. In this work, the information is gathered from the Intel Berkeley Research Laboratory using sensors. Then the Adams-Bashforth-Moulton $(\mathrm{ABM})$ scheme is adopted for information prediction and correction. Basically, $\mathrm{ABM}$ scheme is an iterative multi-step numerical process, i.e., the method uses information from the previous system states. It makes information more accurate and reliable in the sensor node. Finally, simulations results show that the proposed approach provides significant performance improvement compared with the existing approach.

Rest of this paper is organized as follows: Section 2 is about the related works. In Section 3, our proposed method is described. Section 4 deals with algorithm of our proposed method. In Section 5, we discuss the simulation results, and finally, Section 6 concludes the paper.

\section{Related Literature}

One of the most important elements for sensing information is the wireless sensor network. In WSN, bringing down the level of consumed energy is main intention and transmission of data is the prime cause for wireless sensor network to consume energy. When $\mathrm{n}$-bit data is transmitted at a distance begin $R_{c}$, the required energy is defined as follows [1]:

$$
E_{t x}\left(n, R_{c}\right)=E_{\text {elec }} * n+e_{\text {amp }} * n * R_{c}^{2},
$$

where, $E_{\text {elec }}$ is the required energy in the transmission process, $R_{c}$ is the distance and $n$ is the number of bits. The energy required to receive n-bit information is defined as [1]: 


$$
E_{r x}=E_{\text {elec }} * n
$$

In fact, the energy efficiency will increase for WSN because from Equation (2) and Equation (3). We can see that there is a certain amount of energy required to receive and transmit data in sensor networks. To overcome this, data aggregation technique is introduced [3]. Prediction based data collection protocol to eliminate the redundant data transmission was proposed in [4]. Here, sensory data is gathered and routed to cluster head $(\mathrm{CH})$, when their change proceed in definite scheme. Basically, the $\mathrm{CH}$ should predict the future sensory data, rather than using sensory data sensed by sensor nodes in real-time [5]. Both the sink and cluster head predict data using earlier times data. Then a comparison is made between the predicted data and measured data. If the data error is in the range of preconfigured threshold then the data is not sent to the sink, it is discarded by $\mathrm{CH}$. Though this method reduces data transmission rate but it requires synchronization before every transmission, which is a drawback of this method. In [6], it used the Simpson's 3/8 to compute $x(t+1)$ time data by using previous time data. This approach gives good performance when the threshold is high (e.g., 0.21), it can have data prediction accuracy around $25 \%$ but fails to predict data when the threshold is low. In this case, the prediction accuracy may go just around $10 \%$ which is a big drawback of this method.

Mou $\mathrm{Wu}$ et al. [7] proposed a least mean square (LMS) dual prediction algorithm to predict data for sensor network. It performance was better than earlier mentioned methods. It achieves higher accuracy when the prediction threshold is comparatively large. However, it performance is degraded when the prediction threshold is reduced to a smaller value.

Our proposed method has following advantages over previously mentioned methods and they are given as follow:

- The method has higher successful rate of data prediction which minimizes the energy consumption.

- The system can be reconfigured by varying step size parameter. But big step sizes (e.g., $h=0.005$ ) increases the unsuccessful prediction and too small step sizes (e.g. $h<10^{-5}$ ) do not increase the accuracy significantly.

- In proposed algorithm, there are only 5 additions, 3 subtractions and 11 multiplications are required, in total only 19 arithmetic operations are required to predict a sensor value.

\section{Proposed Method}

Generally speaking, the sensor network is divided into centralised, decentralised and hierarchical. In hierarchical sensor network (HSN), sensor nodes are divided into clusters and one node is assigned as the cluster head of the cluster which is responsible for sending data to sink [6] [8] [9]. Each sensor node (SN) sends data to the cluster head $(\mathrm{CH})$ which takes decision about routing data towards the sink based on the prediction accuracy. The base station and sensor nodes form a mesh network in conjunction [8]. 
The target of the HSN architecture is to improve the efficiency by lessening power usage, total dimension and cost loss on the whole. HSN can be kept unaccompanied and remotely controlled. Installation cost and maintenance charge are comparably low and the network can go on for a long time without supervision while providing continual performance [8] [9] [10].

For all sensor nodes which are planted at a distance, only the requisite wireless communication operations are implemented. It means the SN utilizes the single channel to route its data records by broadcasting. To accurately send the results for the sensor nodes to the base station (BS), a CH is introduced in the HSN. In the HSN architecture, each $\mathrm{SN}$ is connected to one and only one $\mathrm{CH}$ and then the whole network is partitioned into several clusters. Inside each cluster, the $\mathrm{CH}$ makes use of a centralized communication protocol to exchange data with its sensor nodes and then transmits the detection records received from its sensor nodes to the base station. This method partitions the entire sensor network into a many compact networks and so decreases the communication complexity of the system in a great deal for both the sensor nodes and the CHs.

HSNs have many important applications such as health care monitoring, air quality monitoring, battlefield surveillance, water purity monitoring, environmental/earth sensing, industrial/machine health study, structural health monitoring [9]. By planting sensor nodes in a battlefield, the situation of territory, routes, paths, and inlets, the actions of the hostile party can be observed from a central control unit remotely. In health monitoring, possible applications include body status measurement, location, monitoring of ill patients (heart rate and blood pressure) in hospitals and at homes [10]. Cluster heads can predict sensor data by applying data aggression by ABM's method [2] [11] [12] [13] [14] [15] before transmitting the collected data towards the sink node and can reduce data transmission between cluster head and sink node. Therefore, this approach not only minimizes the complexity of the sensor nodes implementation but also reduces the cost, total dimensions and power usage of each sensor node remarkably [7] [12] [16] [17] [18] [19]. In our earlier work [16], we tested our method for a single sensor with same scenario. Basically, the single sensor sends information to the cluster head and the cluster head $(\mathrm{CH})$ makes the decision about sending or not sending data to the base station. In practical case, there are more than one sensor connected to the cluster head. So, we extended our work for a large scale sensor networks. For doing this, we are increasing the number of sensors from one to seven with two cluster heads. The information was collected from Intel Barkley Research Lab Data [14]. Figure 1 represents the data fusion in HSN. Epoch 1, 2, 3, 4, 5, 6 and 7 are under $\mathrm{CH}-1$ and labeled as $(1,1),(1,2),(1,3), \cdots,(1,7)$. Epoch $8,9,10,11,12,13,14$ are under $\mathrm{CH}-2$ and labeled as $(2,1),(2,2), \cdots,(2,7)$, respectively.

In the proposed method, the cluster head tries to pre-determine the sensor data using ABM method [2] [11]. The data prediction is done by [2] [11]:

$$
y_{i+1}=y_{i}+\frac{h}{24}\left(55 f_{i}-59 f_{i-1}+37 f_{i-2}-9 f_{i-3}\right) \text {. }
$$




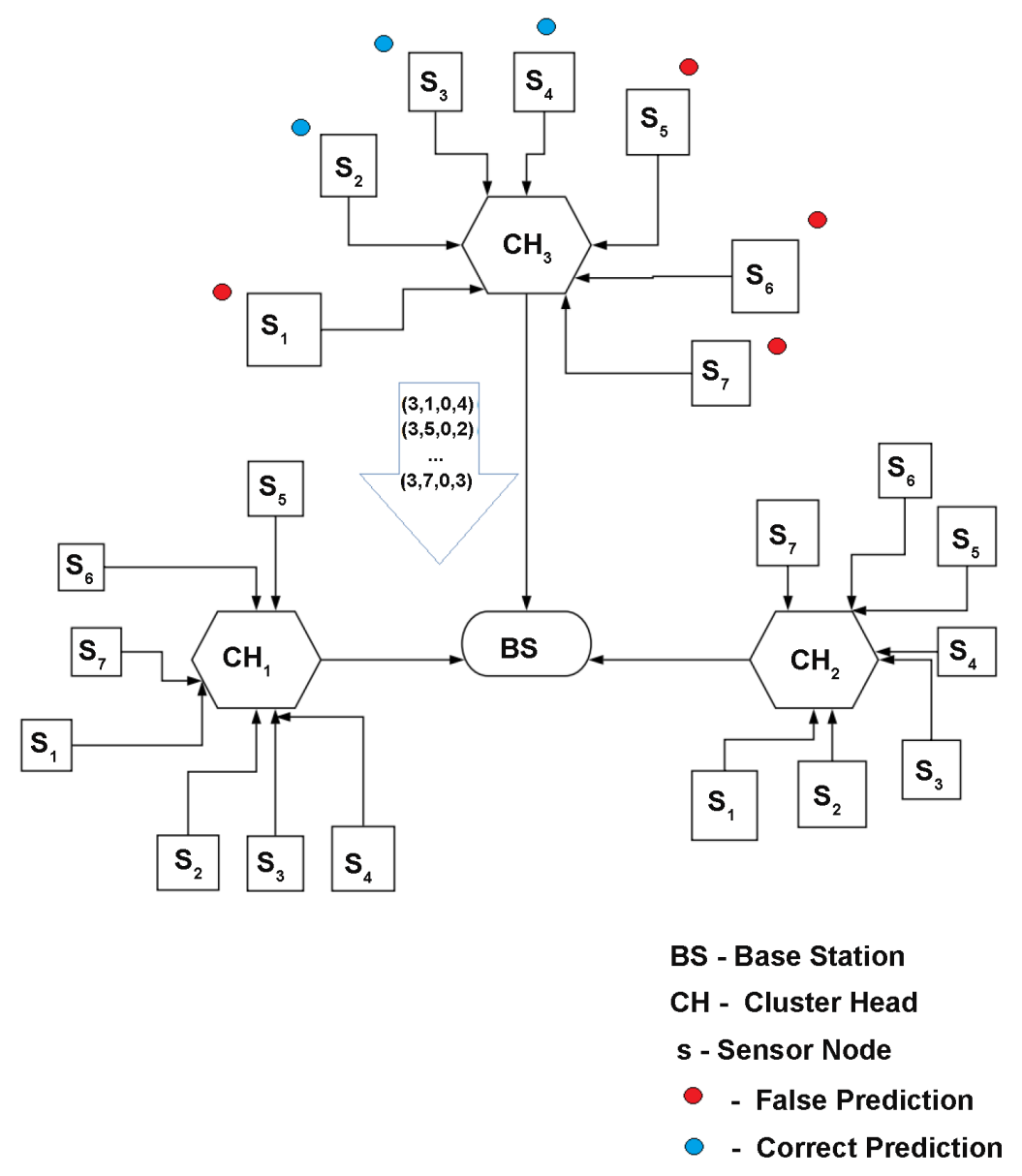

Figure 1. Sensor network based architecture.

and

$$
y_{i+1}=y_{i}+\frac{h}{24}\left(f_{i-2}+5 f_{i-1}+19 f_{i}+9 f_{i+1}\right) \text {. }
$$

Here, Equation (4) is the Adams Bashforth predictor formula [2] and Equation (5) is the Adams Moulton corrector formula [2]. If the difference between the predicted data and actual sensor data is within prediction threshold then data is not transmitted by cluster head and base station uses the predicted data generated by it. If the difference is more than prediction threshold the data is sent to base station. Epoch positions are shown in Figure 2 [14].

Data was collected from 28th February to 5th April, 2004. Mica2Dot sensors with weather boards collected timestamped topology information, along with humidity, temperature, light and voltage values once every 31 seconds. Data was collected using the TinyDB in-network query processing system, built on the TinyOS platform [14].

Data prediction for each epoch or sensor were done separately. Although data for humidity, temperature, light and voltage values were available in the data set but we chose to work with voltage values. When the sensor sends the value of reading to the $\mathrm{CH}$, the $\mathrm{CH}$ predicts the value using four previous time value and 


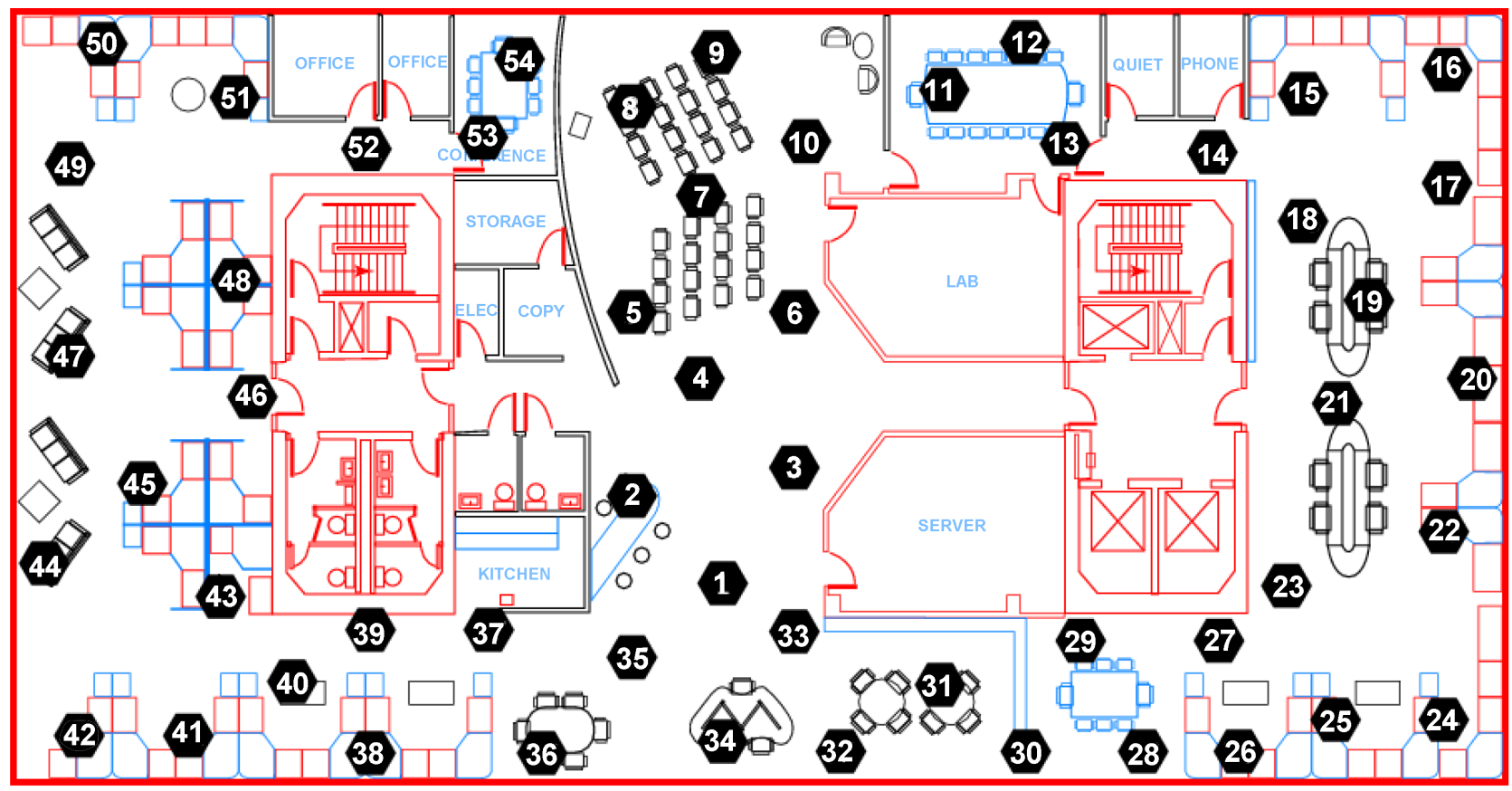

Figure 2. Sensor distribution in intel barkley research lab [14].

compares the predicted value with the received value. If the absolute difference between these two values is within the threshold level, then the $\mathrm{CH}$ discards the data as the base station also predicts the data. If not, then the difference between actual data and predicted data is transmitted to base station.

While sending the data, $\mathrm{CH}$ sends data into a packet. Each packet contains one or more than one data depending on the predicted and actual result. Therefore, to interpret the information correctly another scheme is used. When the $\mathrm{CH}$ sends a packet, every tuple has three parts, i.e. $\mathrm{CH}$ number-epoch id difference of two data, e.g. 2-3-0.24. Finally, base station receives the packet and adds 0.24 to the predicted data for 3 rd epoch of 2 nd cluster.

\section{Proposed Method}

After obtaining information from the sensors, the proposed algorithm is predicted information. Then it follows the correction step and compared with the threshold value. Based on the threshold, the base station is decided the information is transmitted or not. In this way, the energy is save in sensor nodes. In short, the step-by-step process is illustrated in the followings:

INPUT:

$y_{i-3}, y_{i-2}, y_{i-1}, y_{i} \rightarrow 4$ sensor data from previous time slot. OUTPUT:

$f_{i+1} \rightarrow$ predicted data.

PROCESS:

STEP 1: Take 4 earlier time sensor data $y_{i-3}, y_{i-2}, y_{i-1}, y_{i}$ STEP 2: Compute the prediction using Adams-Bashforth predictor equation: 


$$
y_{i+1}=y_{i}+\frac{h}{24}\left(55 f_{i}-59 f_{i-1}+37 f_{i-2}-9 f_{i-3}\right) .
$$

STEP 3: Correct the prediction by Adams-Moulton corrector equation:

$$
y_{i+1}=y_{i}+\frac{h}{24}\left(f_{i-2}+5 f_{i-1}+19 f_{i}+9 f_{i+1}\right) .
$$

STEP 4: At time $\mathrm{i}$, the cluster head computes $y_{i+1}$

STEP 5: Then, cluster head receives $g_{i+1}$ from sensor nodes.

STEP 6: If $|f(i+1)-g(i+1)|<\epsilon$

Transmission is not required.

Else:

Transmission is required.

STEP 7: Update new sensor values.

\section{Simulation Results and Discussions}

For simulation, we have chosen 100 data sample for epoch no 1 to 14 excluding epoch 5. For epoch 5, we only took 35 sample data. Because some data points were missing. The sample data for epoch $(1,3)$ is shown in Table 1 . We have chosen the threshold value to be 0.01 , which is very small threshold. The simulation results are shown in Table 2.

There is an advantage of not sending actual data. Because actual data representation requires more number of bits than difference of actual and predicted data. As the cluster head also predicts the data, then adding the difference to the predicted data results in the original data but energy efficiency is enhanced. From the table below, we can see that ABM method's performance is better than Milne-Simpsons scheme.

We can see that for epoch $(1,5)$ ABM performs very poorly, but Milne-Simpsons method performs moderately. This is because the data points were insufficient. When the data is not sufficient, the Milne-Simpsons method outperforms the ABM method. Cluster heads can predict sensor data by applying data aggression by ABM method more accurately compared with the Simpson's 3/8 and Kalman filter [15] before sending the collected data towards the base station and can decrease data transmission and power usage. This approach significantly reduces the total size, power consumption of the sensor network and cost. Hence, it increases overall network lifetime and efficiency. Overall, it can be seen that the proposed method provides significant performance improvement compared with the existing approach. Therefore, the presented approach is valuable to design the wireless sensor network for prediction and correction the sensing information to save energy.

\section{Conclusion and Future Work}

The energy saving is one of the main tasks in the wireless sensor network. In order to transmit the sensing information to the base station, the sensor node 
Table 1. Sample data for epoch $(1,3)$ for ABM method.

\begin{tabular}{cccc}
\hline Received Data $(\mathrm{X})$ & Predicted Data $(\mathrm{Y})$ & Difference $(\mathrm{Y}-\mathrm{X})$ & Decision \\
\hline 2.19159 & 2.19159 & $2.125 \mathrm{e}-009$ & $\mathrm{NO}$ \\
2.18353 & 2.19159 & 0.00806 & $\mathrm{NO}$ \\
2.19159 & 2.18353 & 0.00806 & $\mathrm{NO}$ \\
2.19159 & 2.19159 & $2.19327 \mathrm{e}-009$ & $\mathrm{NO}$ \\
2.50599 & 2.19159 & 0.3144 & $(1,3,0.31)$ \\
2.50599 & 2.50599 & $2.55839 \mathrm{e}-009$ & NO \\
\hline
\end{tabular}

Table 2. Overall performance of comparison between ABM method and Milne-Simpsons Scheme.

\begin{tabular}{|c|c|c|c|c|c|}
\hline \multicolumn{2}{|c|}{ Sensor Number } & \multicolumn{2}{|c|}{ Prediction Accuracy (\%) } & \multicolumn{2}{|c|}{ Average Prediction Accuracy (\%) } \\
\hline Cluster No. & Sensor No. & $\begin{array}{l}\text { Milne Simpsons } \\
\text { Method }\end{array}$ & ABM Method & $\begin{array}{l}\text { Milne Simpsons } \\
\text { Method }\end{array}$ & ABM Method \\
\hline & 1 & 44.79 & 62.5 & & \\
\hline & 2 & 43.75 & 63.54 & & \\
\hline & 3 & 46.87 & 36.45 & & \\
\hline \multirow[t]{7}{*}{1} & 4 & 63.54 & 63.54 & 55.6471 & 55.6657 \\
\hline & 5 & 32.25 & 3.22 & & \\
\hline & 6 & 62.5 & 63.54 & & \\
\hline & 7 & 95.83 & 96.87 & & \\
\hline & 1 & 43.75 & 54.16 & & \\
\hline & 2 & 47.91 & 59.37 & & \\
\hline & 3 & 76.04 & 77.08 & & \\
\hline \multirow[t]{4}{*}{2} & 4 & 62.5 & 66.66 & 62.7957 & 64.8757 \\
\hline & 5 & 81.25 & 80.2 & & \\
\hline & 6 & 52.08 & 47.91 & & \\
\hline & 7 & 76.04 & 68.75 & & \\
\hline
\end{tabular}

needs to decide whether information is transmitted or not based on the prediction value. For accurate prediction and correction, the ABM approach is proposed and verified using the information that is gathered from the Intel Berkeley Research Laboratory.

Basically, the proposed method improves the accuracy of data prediction compared with the Simpsons 3/8 method and Kalman filter. The accuracy can be improved by tuning the value of step size with the ABM correction approach. These are the advantages of the proposed method over existing approaches. Future research involves consideration of fading channel in the distributed sensor networks [20] [21]. Designing the appropriate threshold is one of our future works [22]. 


\section{Acknowledgements}

The authors would like to thank the anonymous reviewers. Authors also thank to the Department of Electronics and Communication Engineering, Khulna University of Engineering \& Technology to support. Note that the first two authors conducted simulations and wrote the whole paper, whereas the last two authors supervised them. Note that part of this research [17] was published in the International Conference on Advances in Electrical Engineering, Sept. 2017.

\section{References}

[1] Borg, E., Fichtelmann, B. and Asche, H. (2011) Assessment for Remote Sensing Data: Accuracy of Interactive Data Quality Interpretation. Lecture Notes in Computer Science, Vol. 6783, Springer, Berlin.

[2] Balagurusamy, E. (2005) Numerical Solution of Ordinary Differential Equations. Tata McGraw-Hill Publishing Company Limited, India.

[3] Tan, H.Ö., et al. (2003) Power Efficient Data Gathering and Aggregation in Wireless Sensor Networks. SIGMOD Record, 32, 66-71. https://doi.org/10.1145/959060.959072

[4] Wei, G., Ling, Y., Guo, B., Xiao, B. and Vasilakos, A.V. (2011) Prediction-Based Data Aggregation in Wireless Sensor Networks: Combining Grey Model and Kalman Filter. Journal of Computer Communication, 34, 793-802. https://doi.org/10.1016/j.comcom.2010.10.003

[5] Hou, Z., Fang, W., Niu, J., Shu, L. and Mukherjee, M. (2018) Energy-Efficient Event Determination in Underwater WSNs Leveraging Practical Data Prediction. IEEE Transactions on Industrial Informatics. (In Press)

[6] Rajesh, G., Vinayagasundaram, B. and and Moorthy, G.S. (2014) Data Fusion in Wireless Sensor Network Using Simpson's 3/8 Rule. International Conference on Recent Trends in Information Technology, Chennai, 10-12 April 2014.

[7] Wu, M., Tan, L. and Xiong, N. (2016) Data Prediction, Compression, and Recovery in Clustered Wireless Sensor Networks for Environmental Monitoring Applications. Information Sciences, 329, 800-818.

[8] Ahmed, M.M., Pothalaiah, S. and Rao, D.S. (2016) Real-Time Monitoring of Partially Stable Slopes for Landslide Prediction by Using Wireless Sensor Networks. International Conference on Green Engineering and Technologies, Coimbatore, 19 November 2016. https://doi.org/10.1109/GET.2016.7916638

[9] https://en.wikipedia.org/wiki/Wireless_sensor_network

[10] Zhu, C., Wang, H., Liu, X., Shu, L., Yang, L.T. and Leung, V.C. (2016) A Novel Sensory Data Processing Framework to Integrate Sensor Networks with Mobile Cloud. IEEE Systems Journal, 10, 1125-1136.

[11] Milne, W.E. (1926) Numerical Integration of Ordinary Differential Equations. The American Mathematical Monthly, 33, 455-460. https://doi.org/10.1080/00029890.1926.11986619

[12] Jin, M.H., Wu, W.J., Chen, C.K., Chen, Y.F., Wen, C.M., Kao, C.Y., Yu, S.A., Lin, Y.H., Huang, J.G., Rao, H. and Hsu, C.H. (2004) Hierarchical Sensor Network Architecture for Stationary Smart Node Supervision. Proceedings of SPIE, Smart Structures and Materials 2004: Smart Electronics, MEMS, BioMEMS, and Nanotechnology, 5389, $11 \mathrm{p}$.

[13] https://www.mandargogate.com/comparison-study-clustering-protocols-wsn 
[14] http://db.csail.mit.edu/labdata/labdata.html

[15] Ci, S. and Sharif, H. (2005) Performance Comparison of Kalman Filter Based Approaches for Energy Efficiency in Wireless Sensor Networks. Proceedings of the 3 rd ACS/ IEEE International Conference on Computer Systems and Applications, Cairo, 6 January 2005.

[16] Islam, M.M., Nazi, Z.A, Rana, M.M. and Hossain, A.A.B.M. (2017) Information Prediction in Sensor Networks Using Milne-Simpson's Scheme. Proceedings of the International Conference on Advances in Electrical Engineering, September 2017, 494-498.

[17] Ojha, T., Misra, S. and Raghuwanshi, N.S. (2015) Wireless Sensor Networks for Agriculture: The State-of-the-Art in Practice and Future Challenges. Computers and Electronics in Agriculture, 118, 66-84.

https://doi.org/10.1016/j.compag.2015.08.011

[18] Zhu, C., Leung, V.C., Yang, L.T. and Shu, L. (2015) Collaborative Location-Based Sleep Scheduling for Wireless Sensor Networks Integrated with Mobile Cloud Computing. IEEE Transactions on Computers, 64, 1844-1856.

https://doi.org/10.1109/TC.2014.2349524

[19] Zhu, C., Sheng, Z., Leung, V.C., Shu, L. and Yang, L.T. (2015) Toward Offering More Useful Data Reliably to Mobile Cloud from Wireless Sensor Network. IEEE Transactions on Emerging Topics in Computing, 3, 84-94. https://doi.org/10.1109/TETC.2014.2364921

[20] Xu, Y., Winter, J. and Lee, W.C. (2004) Prediction-Based Strategies for Energy Saving in Object Tracking Sensor Networks. Proceedings of the International Conference on Mobile Data Management, 19-22 January 2004, 346-357.

[21] Zhang, Y., Meratnia, N. and Havinga, P. (2010) Outlier Detection Techniques for Wireless Sensor Networks: A Survey. IEEE Communications Surveys and Tutorials, 12, 159-170. https://doi.org/10.1109/SURV.2010.021510.00088

[22] Bourke, A.K. and Lyons, G.M. (2008) A Threshold-Based Fall-Detection Algorithm Using a Bi-Axial Gyroscope Sensor. Medical Engineering and Physics, 30, 84-90. https://doi.org/10.1016/j.medengphy.2006.12.001 